\title{
Avaliação metabólica do uso de probiótico ou monensina em cordeiros mantidos em semi-confinamento
}

\section{Metabolic assessment of the use of probiotic or monensin in lambs kept in semi-confined conditions}

\author{
Vinicius Coitinho Tabeleão ${ }^{1 *}$; Elizabeth Schwegler' ${ }^{2}$ Sandra Vieira de Moura3 \\ Maikel Alan Goulart ${ }^{4}$; Mariane Aline Weiser ${ }^{5}$; Viviane Maciel da Silva ${ }^{6}$; \\ Talita Bandeira Roos ${ }^{7}$; Francisco Augusto Burket Del Pino ${ }^{8}$; Carlos Gil-Turnes ${ }^{9}$; \\ Cassio Cassal Braunes ${ }^{10}$; Marcio Nunes Corrêa ${ }^{10}$
}

\section{Resumo}

O objetivo do estudo foi verificar o efeito de probióticos e monensina sobre parâmetros metabólicos de cordeiros desmamados. No primeiro experimento foram utilizados 30 cordeiros, alimentados durante 6 semanas, de acordo com os seguintes tratamentos. GC1: Controle (sem aditivo), GB1: Bacillus cereus var. toyoi $\left(1 \times 10^{6}\right.$ esporos/g) e GS1: Saccharomyces boulardii $\left(1 \times 10^{6}\right.$ células viáveis/g). No segundo experimento foram utilizados 30 cordeiros, alimentados durante 8 semanas divididos em três tratamentos: GC2: Controle (sem aditivo), GM2: Monensina sódica (500g/t) e o GS2: Saccharomyces boulardii ( $1 \times 10^{6}$ células viáveis/gr). Semanalmente foram coletas amostras de sangue. Em ambos os experimentos foram avaliados: glucose, triacilglicerídeos (TAG), colesterol, albumina, ureia, aspartato amino transferase (AST), creatinina quinase (CK) e gama glutamil transferase (GGT). O GS1 obteve maiores concentrações de albumina e ureia $(\mathrm{P}<0,01)$, quando comparado aos demais grupos, no período adaptativo. O GC1 demonstrou maiores concentrações de colesterol, albumina e atividade de AST $(\mathrm{P}<0,05)$, e menores de glucose, ureia e atividade de CK $(\mathrm{P}<0,05)$, quando comparadas ao GS1, no período pós-adaptativo. No experimento dois durante o período adaptativo o GS2 obteve maiores concentrações de albumina e ureia $(\mathrm{P}<0,01)$, quando comparado aos demais. O GM2 obteve maiores concentrações de TAG $(\mathrm{P}<0,01)$ e menores de glucose e atividade de $\mathrm{CK}(\mathrm{P}<0,05)$, quando comparado aos demais, no período adaptativo. O GS2 obteve menores concentrações de colesterol e atividade de GGT $(\mathrm{P}<0,05)$, quando comparado com os demais, no período pós-adaptativo. O GC2 obteve maiores concentração de albumina e atividade de AST $(\mathrm{P}<0,01)$, e menores de ureia $(\mathrm{P}<0,05)$, quando comparados com os demais, no período pós-adaptativo. O GM2 obteve maior atividade de

\footnotetext{
${ }^{1}$ Médico Veterinário, Discente de Doutorado em Biotecnologia, Universidade Federal de Pelotas, UFPel, Pelotas, RS. E-mail: tabeleao@bol.com.br

${ }^{2}$ Médica Veterinária, Prof ${ }^{a}$ de Produção Animal, Instituto Federal Catarinense, Campus Araquari, Araquari, E-mail: bethveterinaria@ gmail.com

${ }^{3}$ Médica Veterinária, Discente de Doutorado em Veterinária, UFPel, Pelotas, RS. E-mail: sandra.moura.vet@gmail.com

${ }^{4}$ Médico Veterinário, M.e emVeterinária, UFPel, Pelotas, RS. E-mail: maikelalan.goulart@gmail.com

${ }^{5}$ Médica Veterinária, UFPel, Pelotas, RS. E-mail: marianeweiser@hotmail.com

${ }^{6}$ Prof ${ }^{a}$ do Instituto Sul Rio Grandense, Pelotas, RS. E-mail: aprendaquimicaifsul@gmail.com

${ }^{7}$ Médica Veterinária, Dra. Prof ${ }^{\mathrm{a}} \mathrm{Dr}^{\mathrm{a}}$ da Faculdade de Veterinária, Universidade Federal do Pará, UFPA, Campus Castanhal, Castanhal, PA. E-mail: talitaroos@gmail.com

${ }^{8}$ Farmacêutico, Prof. Dr. do Centro de Ciência Químicas, Farmacêuticas e de Alimentos, UFPel, Pelotas, RS. E-mail: fabdelpino@, gmail.com

${ }^{9}$ Médico Veterinário, Dr. Pesquisador, UFPel, Pelotas, RS. E-mail: gil@ufpel.edu.br

${ }^{10}$ Médicos Veterinários, Profs. Drs. Faculdade de Agronomia Eliseu Maciel, UFPel, Pelotas, RS. E-mail: cassiocb@gmail.com; marcio.nunescorrea@gmail.com

* Autor para correspondência
} 
GGT $(\mathrm{P}<0,05)$ e menor atividade de $\mathrm{CK}(\mathrm{P}<0,05)$, quando comparado com os demais, no período pósadaptativo. $\mathrm{O}$ metabolismo proteico dos cordeiros demonstrou melhor desempenho no período préadaptação com Saccharomyces boulardii, já no período pós-adaptativo a monensina apresentou menor catabolismo muscular.

Palavras-chave: Saccharomyces boulardii, Bacillus cereus, monensina, probiótico, metabolismo

\section{Abstract}

The objective of this study was to verify probiotics and monensin effects on metabolic parameters in weaning lambs. In the experiment 1, thirty lambs divided in three groups were fed during 6 weeks. The lambs were enrolled in one of the following treatments: GC1: as a control, GB1: Bacillus cereus var. toyoi $\left(1 \times 10^{6}\right.$ spores/g) and GS1: Saccharomyces boulardii $\left(1 \times 10^{6}\right.$ viable cells/g). In the experiment 2 , thirty lambs divided in three groups were fed during 8 weeks. The treatments consisted in GC2: as a control, GM2: monensin (500g/t) and GS2: Saccharomyces boulardii (1x10 viable cells/g). In both experiments blood samples were collected weekly aiming to measure metabolic parameters as glucose, triacylglycerol (TAG), cholesterol, albumin, urea, aspartate amino transferase (AST), creatinine kinase (CK) and gamma glutamil transferase (GGT). The results from experiment 1 demonstrated that GS1 had greater $(\mathrm{P}<0.05)$ concentrations of albumin and urea during the adaptation period. In the postadaption period the $\mathrm{GC} 1$ demonstrated increased $(\mathrm{P}<0.05)$ concentrations of cholesterol, albumin and AST activity, in contrast, reduced $(\mathrm{P}<0.05)$ concentrations of glucose, urea and $\mathrm{CK}$ in comparison to GS1. However, during experiment two throughout the adaptation period the GS2 had greater $(\mathrm{P}<0.05)$ concentrations for albumin and urea. The GM2 had greater $(\mathrm{P}<0.05)$ concentrations for TAG and lower $(\mathrm{P}<0.05)$ for glucose and CK activity. During the post-adaption period the GS2 showed the lowest $(\mathrm{P}<0.05)$ concentrations for cholesterol and GGT activity, GC2 had greater $(\mathrm{P}<0.05)$ levels of albumin and AST activity as well as lower concentrations for urea. The GM2 had greater levels for GGT activity and lower levels for CK activity. Therefore, it is concluded that protein metabolism in lambs has better performance when Saccharomyces boulardii is added in the ration during the adaptation period. However, during post-adaptation period, monensin is more beneficial for the protein metabolism, once it does not interfere in the muscles catabolism.

Key words: Saccharomyces boulardii, Bacillus cereus, ruminal fluid metabolism

\section{Introdução}

As altas quantidades de concentrados, comumente utilizados na alimentação de ruminantes, estão associadas com enfermidades subclínicas (VAN CLEEF, E. H. et al., 2009). Com intuito de potencializar a produção e diminuir prováveis perdas produtivas são utilizados aditivos à base de antibiótico ionóforo (monensina) em doses subterapêuticas (DOYLE, 2001). Este produto tem por função controlar principalmente as bactérias produtoras de metano, diminuir a desaminação das proteínas e aumentar a digestibilidade de dietas quando ricas em fibras (RODRIGUES et al., 2001; RUSSELL; HOULIHAN, 2003, ROZZA et al., 2007), melhorando o desempenho produtivo. Entretanto, trabalhos apontam que pode ocorrer a intoxicação por monensina de forma acidental, sendo resultado de falhas na preparação das dietas, tais como alta dosagem, erro na diluição da ração, e uso da substância em espécies não incluídas nas recomendações do produto (NOVILLA, 1992, HALL, 2004, NOGUEIRA; FRANÇA; PEIXOTO, 2009). Além disso outro ponto negativo seria o objetivo de ser reduzir a utilização de antibióticos para promoção de sistemas ambientalmente corretos. Além dos fatores citados, esse tipo de aditivo pode deixar resíduos na carne e leite (RUSSELL; HOULIHAN, 2003).

Por estas razões, alguns pesquisadores têm buscado alternativas a este princípio ativo, como a utilização de probióticos à base de bactérias, dentre elas: Lactobacillus sp., Bifidobacteria sp., 
Streptococcus faecium, Enterococcus faecium, Bacillus cereus var. toyoi (COPPOLA; TURNES, 2004, WEINBERG et al., 2004), e/ou leveduras: Saccharomyces cerevisiae, Saccharomyces boulardii, entre outras (ROBINSON; GARRETT, 1999, CZERUCKA et al., 2000). Estes microrganismos probióticos estabelecem competição com a microbiota residente no rúmen ligando-se com as mucosas, bem como são secretores de metabólitos que podem estimular outras colônias benéficas para o organismo hospedeiro (KAMEL et al., 2004; MWENYA et al., 2004), além de poderem ser absorvidos pelos ruminantes, tais como vitaminas do complexo B e peptídeos bioativos (VAN KESSEL; RUSSELL, 1996). Alguns estudos relatam ainda aumento na produção de ácidos graxos voláteis (ENJALBERT et al., 1999), o que é benéfico, pois estes são precursores de compostos energéticos. Apesar de vários estudos tratarem o assunto o impacto da adição de probióticos no ambiente ruminal ainda não está bem esclarecido, bem como os diferentes papéis desses aditivos nos parâmetros metabólicos dos ruminantes. Sendo assim, a hipótese deste estudo é que a substituição da monensina por probióticos é possível sem o comprometimento do metabolismo animal. Desta forma, o objetivo deste trabalho foi verificar a influência de probióticos e monensina sobre parâmetros metabólicos de cordeiros desmamados mantidos em sistema semi-confinado de produção.

\section{Materiais e Métodos}

Os dois experimentos foram realizados no município de Piratini/RS durante a estação de verão, com cordeiros desmamados (75 - 95 dias de vida) mantidos em pastagem nativa sob sistema de semi-confinamento. Estes foram vermifugados com Ivermectina $0,2 \mathrm{mg} / \mathrm{kg}$ no início do experimento e 30 dias após a primeira aplicação. No início do experimento, foi fornecido concentrado Irgovino ${ }^{\circledR}$ IRGOVEL $^{\circledR}$, equivalente a $1 \%$ do peso vivo, sendo divididas duas vezes ao dia, durante cinco dias, com isso adaptando os animais do estudo ao consumo de concentrado.

\section{Animais e desenho experimental \\ Experimento 1}

Foram utilizados 30 ovinos, distribuídos aleatoriamente segundo seu peso inicial e identificados através de brincos auriculares nos grupos experimentais, durante 6 semanas. Cada grupo experimental possuía 5 machos e 5 fêmeas, sendo distribuídos de forma homogênea para compor os seguintes grupos: $\mathbf{G C 1}(\mathrm{n}=10)$ : recebiam ração sem aditivo; GB1 $(\mathrm{n}=10)$ : recebiam ração contendo $1 \times 10^{6}$ esporos de Bacillus cereus var. toyoi por grama de ração; GS1 ( $\mathrm{n}=10)$ : recebiam ração contendo $1 \times 10^{6}$ células viáveis de Saccharomyces boulardii por grama de ração.

\section{Experimento 2}

Foram utilizados 30 ovinos, distribuídos aleatoriamente segundo seu peso inicial e identificados através de brincos auriculares, nos grupos experimentais, durante 8 semanas. Cada grupo experimental possuía 5 machos e 5 fêmeas, sendo distribuídos de forma homogênea para compor os seguintes grupos: GC2 $(\mathrm{n}=10)$ : recebiam ração sem aditivo; GM2 $(\mathrm{n}=10)$ : recebiam ração contendo $500 \mathrm{~g} / \mathrm{t}$ de monensina sódica; GS2 $(\mathrm{n}=10)$ : recebiam ração contendo $1 \times 10^{6}$ células viáveis de Saccharomyces boulardii por grama de ração. Os procedimentos de formulação de dietas, manejo dos animais e coletas de sangue se manteve igual em ambos experimentos.

\section{Formulação de dietas e manejo}

A monensina sódica, bem como o Bacillus cereus var. toyoi foram incorporados às rações através de misturador automático, mantendo-se estas as mesmas durante o período experimental. Inicialmente os aditivos foram adicionados a uma 
pré-mistura com premix mineral e vitamínico. Após esta etapa foi realizada uma mistura aos demais ingredientes da ração, totalizando $1 \mathrm{t}$ de mistura final farelada. Entretanto, o Saccharomyces boulardii foi adicionado através de um processo de mistura manual diariamente em ração farelada, a fim de impedir a germinação das células antes da ingestão da ração. Após o período de adaptação, os ovinos foram separados de acordo com cada tratamento. Logo depois da ingestão completa do concentrado os cordeiros eram soltos em pastagem nativa. A ração comercial fornecida durante o estudo foi Irgovino $^{\circledR}$ IRGOVEL $^{\circledR}$, a qual era composta de $20 \%$ de proteína bruta, $15 \%$ de matéria mineral, $2 \%$ de cálcio, $0,8 \%$ de fósforo, $2 \%$ de extrato etéreo e $12 \%$ matéria fibrosa. Semanalmente, foi ajustada a oferta de concentrado, sendo o equivalente a $1 \%$ do peso vivo.

\section{Coleta de materiais e análises}

A coleta de sangue foi realizada semanalmente em ambos os experimentos, através de punção da veia jugular, sendo divididas alíquotas em três tubos de ensaio, contendo $5 \mathrm{~mL}$ de sangue total em cada frasco. Um dos tubos continha anticoagulante (EDTA 10\%) na proporção de $12 \mu \mathrm{L} / \mathrm{mL}$ de sangue, outro tubo continha EDTA $10 \%$ e inibidor da glicólise (KF 12\%) na proporção de $12 \mu \mathrm{L} / \mathrm{mL}$ e 16 $\mu \mathrm{L} / \mathrm{mL}$ respectivamente de sangue, um terceiro tubo não continha anticoagulante. Posteriormente as amostras foram centrifugadas a $1800 \mathrm{x} g$ durante 10 minutos e divididas em dois tubos tipo eppendorff previamente identificados, dos quais um foi congelado a $-18^{\circ} \mathrm{C}$ e o outro foi resfriado a $4^{\circ} \mathrm{C}$. Os marcadores avaliados nos dois experimentos foram: glucose, triacilgliceróis (TAG), colesterol, albumina, ureia, aspartato amino transferase (AST),creatinina quinase (CK) e gama glutamil transferase (GGT) por métodos colorimétricos usando kits comerciais LABTEST $^{\circledR}($ TABELEÃO et al., 2007).

\section{Análise estatística}

Os resultados são apresentados como médias \pm erro padrão da média (EPM). Todas as análises estatísticas foram realizadas utilizando o software SAS (SAS Institute Inc., Cary, NC, USA). Análises envolvendo medidas repetidas ao longo do tempo dos metabólitos glucose, triacilgliceróis, colesterol total, ureia, albumina, AST, GGT e CK foram comparadas entre tratamentos por meio de análise de variância para medidas repetidas, utilizando o procedimento MIXED para avaliar os principais efeitos do tempo, o tratamento, e suas interações (LITTELL; HENRY; AMMERMAN, 1998). Os modelos estatísticos e as análises de dados foram separados em adaptação (3 semanas iniciais) e pósadaptação (4 a 6 semanas exp. 1 e de 4 a 9 semanas exp. 2). Os valores de $\mathrm{P}<0,05$ foram considerados significativos.

\section{Resultados e Discussão}

Os resultados das médias dos metabólitos do experimento 1 e do experimento 2, bem como suas interações entre tempo e tratamento, além dos efeitos de coletas estão descritos nas tabelas 1 e 2 , respectivamente. 


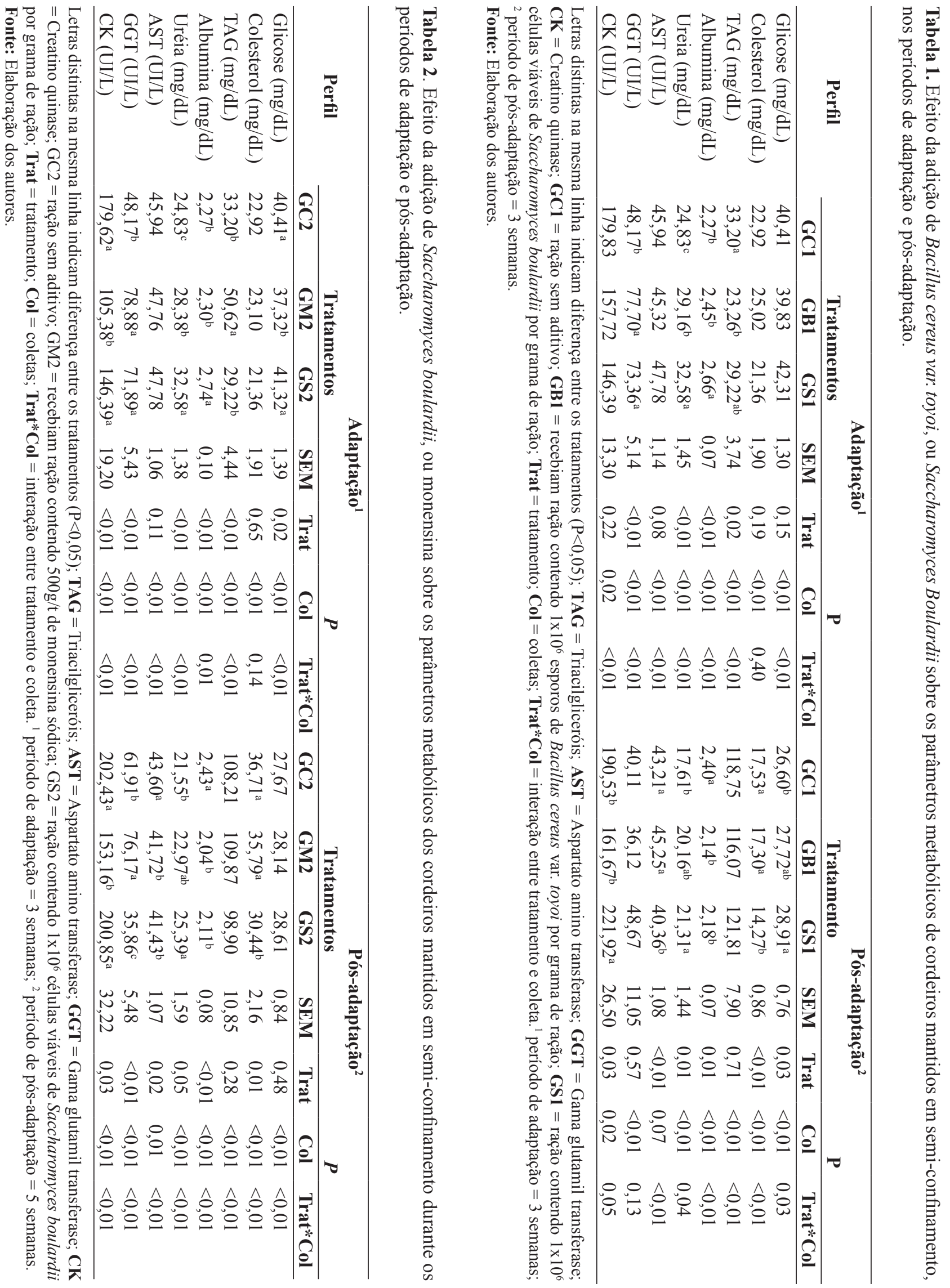


Duranteo período de adaptação, dos experimentos 1 e 2, pôde ser verificado que os animais dos grupos GS1 e GS2 obtiveram maiores concentrações médias plasmáticas de albumina e uréia $(\mathrm{P}<0,01)$, quando comparados aos grupos controles (GC1 e GC2), nos respectivos experimentos. Este fato esta provavelmente relacionado com a adição do Saccharomyces boulardii que alterou o ambiente ruminal e propiciou maior crescimento da microbiota residente, e desta forma gerou maior quantidade de proteína microbiana para o duodeno (ROBINSON; GARRETT, 1999), já que o suplemento fornecido era isoprotéico. Este incremento protéico pode ser confirmado indiretamente através do aumento das concentrações plasmáticas de ureia $(\mathrm{P}<0,05)$, que pode ter ocorrido devido ao aumento no ciclo de catabolismo protéico hepático, ou ainda pelo incrementado a produção de nitrogênio ruminal (WILLIAMS et al., 2004).

No que se refere à concentração plasmática média de TAG, durante o período de adaptação, pode ser verificado que o grupo GB1 demonstrou concentração inferior $(\mathrm{P}<0,05)$ ao grupo $\mathrm{GC1}$, no entanto, similar ao grupo GS1. Contudo, no experimento 2, o grupo GM2 apresentou maior média na concentração de TAG $(\mathrm{P}<0,01)$ e menor de glucose $(\mathrm{P}<0,05)$, comparados aos demais grupos do experimento 2. Sendo assim, pode-se verificar que estes aditivos modificaram a produção de ácidos graxos voláteis no rúmen, entretanto, mantendose dentro da faixa fisiológica. Uma vez que estes ácidos graxos, são utilizados como substratos para a síntese de triacilgliceróis e glucose (RIBEIRO et al., 2004, BAL1KC1; Y1LD1Z; GÜRDOĞAN, 2007). Além disso, a média de atividade da enzima $\mathrm{CK}$ foi menor $(\mathrm{P}<0,01)$ no grupo GM2, indicando menor utilização desse tecido como fonte de proteína, uma vez que este tecido é importante reserva de aminoácidos para o organismo (SOBIECH; KULETA, 2002). Desta forma, este comportamento foi importante, já que esta enzima pode ser utilizdo como marcador de subnutrição, contudo os níveis de variação estão dentro da faixa fisiológica.
A inclusão dos aditivos nos grupos GB1, GS1, GM2 e GS2 promoveram alterações na funcionalidade hepática, no período de adaptação, que foram evidenciadas pela maior $(\mathrm{P}<0,01)$ atividade da enzima GGT, quando comparadas com os controles (GC1 e GC2) dos respectivos experimentos, já que este marcador é sensível a alterações de atividade dos hepatócitos (HATFIELD et al., 1998, MELAKU; PETERS; TEGEGNE, 2004). Contudo, as concentrações média dos marcadores de glucose, colesterol, AST e CK, mantiveram-se semelhantes $(\mathrm{P}>0,05)$ entre os grupos do experimento 1, assim como as concentrações plasmáticas médias de colesterol e a atividade média da AST do experimento 2, no período de adaptação, não demonstrando alterações tecidual, e portanto, demonstrando que os animais mantiveram o mesmo padrão de saúde.

Após o período de adaptação da microbiota ruminal, pode-se verificar que os animais dos grupos GS1 e GS2 apresentaram menores concentrações plasmáticas médias de colesterol $(\mathrm{P}<0,01)$ que os demais grupos em ambos os experimentos. Este evento pode estar relacionado com a atividade anticolesterolêmica, provocado pelas leveduras do gênero Saccharomyces (MOUSA et al., 2012), já que as concentrações médias plasmáticas de triacilgliceróis, que utilizariam o colesterol como parte das moléculas transportadoras (LUBBADEH et al., 1999) não diferiam $(\mathrm{P}>0,05)$ entre os tratamentos no experimento 1 e 2 . No entanto, no experimento 1 o grupo GS1 pode ter sofrido alteração na produção de ácidos graxos voláteis e com isso causando o aumento na neoglicogênese (MOLONEY; ALLEN; ENRIGHT, 2002), já que as concentrações plasmáticas médias deste metabólito estavam maiores $(\mathrm{P}<0,05)$, quando comparados ao grupo GC1. Entretanto, o mesmo fato não foi observado no experimento $2(\mathrm{P}>0,05)$. Estas alterações constatadas, não devem comprometer a funcionalidade dos órgãos causando alguma disfunção tecidual, já que a amplitude de variação foi pequena, embora tenha sido estatística. 
Em relação ao metabolismo proteico, no que se refere à concentração média plasmática de albumina, no período pós-adaptativo foi observado que os grupos contendo aditivos (GB1, GS1, GM2 e GS2) obtiveram níveis menores $(\mathrm{P}<0,05)$ que seus respectivos controles (GC1 e GC2), em ambos os experimentos. Isto denota que os aditivos não foram capazes de modificar a microbiota ruminal de forma que pudesse interferir no fornecimento de aminoácidos para a síntese de albumina (RIBEIRO et al., 2004). Contudo, a presença do Saccharomyces boulardii (GS1 e GS2) pode ter promovido incremento $(\mathrm{P}<0,05)$ nas concentrações médias plasmáticas de ureia, quando comparados aos grupos GC1 e GC2, respectivamente. Este fato sugere que o microorganismo pode ter alterado a metabolização de proteínas no rúmen e com isso aumentando a concentração de nitrogênio intraruminal, e desta forma aumentando a concentração plasmática de ureia (RIBEIRO et al., 2004, WILLIAMS et al., 2004).

Esta alteração do metabolismo runinal gerou menor aporte proteico para absorção duodenal, e consequentemente influenciando a menor $(\mathrm{P}<0,05)$ atividade da enzima AST em ambos os experimentos dos grupos GS1 e GS2, assim como no grupo GM2, desta forma diminuindo a atividade da rota de desaminação e com isso reduzindo a atividade da enzima AST (SOBIECH; KULETA, 2002, SRIKANDAKUMAR; JOHNSON; MAHGOUB, 2003). No mesmo sentido, observa-se que o grupo GS1 obteve maior $(\mathrm{P}<0,05)$ média de atividade da enzima CK, demonstrando-se que pode ter sido utilizado a reserva muscular, a fim de suprir a deficiência protéica. Porém, a atividade média de $\mathrm{CK}$ foi menor $(\mathrm{P}<0,05)$ no grupo GM2, quando comparados aos grupos GC2 e GS2, o que pode ter demonstrado menor atividade de catabolismo muscular (SOBIECH; KULETA, 2002).

Durante o período de pós-adaptativo do experimento 2, o GM2 pode ter gerado alteração na funcionalidade hepática, já que a atividade média da enzima GGT foi maior $(\mathrm{P}<0,01)$, sugerindo que o aditivo tenha desenvolvido efeito tóxico nestas concentrações, além do que, os demais metabólitos avaliados não demonstraram evidências que induzissem este maior funcionamento hepático (MELAKU; PETERS; TEGEGNE, 2004, MEIRAJUNIOR et al., 2009).

\section{Conclusão}

A inclusão de probióticos e monensina em dietas de cordeiros demonstra ter efeitos semelhantes sobre parâmetros metabólicos. Porém, o metabolismo proteico dos cordeiros apresenta melhor desempenho no período pré-adaptação, quando Saccharomyces boulardii é utilizado na dieta. Já no período pós-adaptativo a utilização de monensina apresenta melhores efeitos no metabolismo proteico verificada pela menor catabolismo muscular.

\section{Referências}

BAL1KC1, E.; Y1LD1Z, A.; GÜRDOĞAN, F. Blood metabolite concentrations during pregnancy and postpartum in Akkaraman ewes. Small Ruminant Research, Amsterdam, v. 67, n. 2-3, p. 247-251, 2007.

COPPOLA, M. D. M.; TURNES, C. G. Probióticos e resposta imune. Ciência Rural, Santa Marria, v. 34, n. 4, p. 1297-1303, 2004.

CZERUCKA, D. S.; DAHAN, B.; MOGRABI, B.; ROSSI, RAMPAL, P. Saccharomyces boulardii preserves the barrier function and modulates the signal transduction pathway induced in enteropathogenic Escherichia coliinfected T84 cells. Infection and Immunity, Washington, v. 68, n. 10, p. 5998-6004, 2000.

DOYLE, M. E. Alternatives to antibiotic use for growth promotion in animal husbandry. Food Research Institute Briefings, Madison, p. 1-17, 2001. Disponível em: $<$ https://fri.wisc.edu/docs/pdf/antibiot.pdf $>$. Acesso em: 18 jul. 2009.

ENJALBERT, F. J.; GARRETT, E.; MONCOULON, R.; BAYOURTHE, C.; CHICOTEAU, P. Effects of yeast culture (Saccharomyces cerevisiae) on ruminal digestion in non-lactating dairy cows. Animal Feed Science and Technology, Amsterdam, v. 76, n. 3-4, p. 195-206, 1999. 
HALL, J. H. Ionophores. In: PLUMLEE, E. B. Clinical veterinary toxicology. Mosby: St. Louis, Missouri, 2004. p. $120-127$.

HATFIELD, P. G.; HOPKINS, J. A.; RAMSEY, W. S.; GILMORE, A. Effects of level of protein and type of molasses on digesta kinetics and blood metabolites in sheep. Small Ruminant Research, Amsterdam, v. 28, n. 2, p. 161-170, 1998.

KAMEL, H. E. M.; SEKINE, J.; EL-WAZIRY, A. M.; YACOUT, M. H. M. Effect of Saccharomyces cerevisiae on the synchronization of organic matter and nitrogen 62 degradation kinetics and microbial nitrogen synthesis in sheep fed Berseem hay (Trifolium alexandrinum). Small Ruminant Research, Amsterdam, v. 52, p. 211-216, 2004.

LITTELL, R. C.; HENRY, P. R.; AMMERMAN, C. B. Statistical analysis of repeated measures data using SAS procedures. Journal of Animal Science, Stanford, v. 76, n. 4, p. 1216-1231, 1998.

LUBBADEH, W.; HADDADIN, M. S. Y.; AL-TAMIMI, M. A.; ROBINSON, R. K. Effect on the cholesterol content of fresh lamb of supplementing the feed of Awassi ewes and lambs with Lactobacillus acidophilus. Meat Science, Champaign, v. 52, n. 4, p. 381-385, 1999.

MEIRA-JUNIOR, E. B. D. S.; RIZZO, H.; BENESI, F. J.; GREGORY, L. Influência dos fatores sexuais e etários sobre a proteína total, fração albumina e atividade sérica de aspartato-aminotransferase e gama-glutamiltransferase de ovinos da raça Santa Inês. Brazilian Journal of Veterinary Research and Animal Science, São Paulo, v. 46, n. 6, p. 448-454, 2009.

MELAKU, S.; PETERS, K. J.; TEGEGNE, A. Feed intake, live weight gain and reproductive performance of Menz ewes supplemented with Lablab purpureus, graded levels of Leucaena pallida 14203 and Sesbania sesban 1198. Livestock Production Science, Amsterdam, v. 87, n. 2-3, p.131-142, 2004.

MOLONEY, A. P.; ALLEN, P.; ENRIGHT, W. J. Body composition and adipose tissue accretion in lambs passively immunised against adipose tissue. Livestock Production Science, Amsterdam, v. 74, n. 2, p. 165-174, 2002.

MOUSA, K. M.; EL-MALKY, O. M.; KOMONNA, O. F.; RASHWAN, S. E. Effect of some yeast and minerals on the productive and reproductive performance in ruminants. Journal of American Science, Richmond Hill, v. 8, n. 2, p. 291-303, 2012.
MWENYA, B.; SANTOSO, B.; SAR, C.; GAMO, Y.; KOBAYASKI, T.; ARAI, I.; TAKAHASKI, J. Effect of including $\beta$ 1-4 galacto-oligosaccharides, lactic acid bacteria or yeast culture on methanogenesis as well as energy and nitrogen metabolism in sheep. Animal Feed Science and Technology, Amsterdam, v. 115, n. 3-4, p. 313-326, 2004.

NOGUEIRA, V. A. N.; FRANÇA, T. N. F.; PEIXOTO, P. V. Intoxicação por antibióticos ionóforos em animais. Pesquisa Veterinária Brasileira, Seropédica, v. 29, n. 3, p. 191-197, 2009.

NOVILLA, M. N. The veterinary importance of the toxic syndrome induced by ionophores. Veterinary \& Human Toxicology, New Jersey, v. 34, n. 1, p. 66-70, 1992.

RIBEIRO, L. A. O.; MATTOS, R. C.; GONZALEZ, F. H. D.; WALD, V. B.; SILVA, M. A. D.; ROSA, V. L. L. Perfil metabólico de ovelhas Border Leicester x Texel durante a gestação e a lactação. Revista Portuguesa de Ciências Veterinárias, Lisboa, v. 99, n. 551, p. 155-159, 2004.

ROBINSON, P. H.; GARRETT, J. E. Effect of yeast culture (Saccharomyces cerevisiae) on adaptation of cows to postpartum diets and on lactational performance. Journal Animal Science, Champaign, v. 77, n. 4, p. 988999, 1999.

RODRIGUES, P. H. M.; MATTOS, W. R. S.; MELOTTI, L.; RODRIGUES, R. R. Monensina e digestibilidade aparente em ovinos alimentados com proporções de volumoso/concentrado. Scientia Agricola, Piracicaba, v. 58, n. 3, p. 449-455, 2001.

ROZZA, D. B.; CORREAA, A. M. R.; LEAL, J. S.; BANDARRA, P. M.; GUAGNINI, F. S.; RAYMUNDO, D. L.; DRIEMEIER, D. Intoxicação experimental por monensina em búfalos e bovinos. Pesquisa Veterinária Brasileira, Seropédica, v. 27, n. 4, p. 172-178, 2007.

RUSSELL, J. B.; HOULIHAN, A. J. Ionophore resistance of ruminal bacteria and its potential impact on human health1. FEMS Microbiology Reviews, Dubrovnik, v. 27, n. 1, p. 65-74, 2003.

SOBIECH, P.; KULETA, Z. Usefulness of some biochemical indicators in detection of early stages of nutritional muscular dystrophy in lambs. Small Ruminant Research, Amsterdam, v. 45, n. 2, p. 209-215, 2002.

SRIKANDAKUMAR, A.; JOHNSON, E. H.; MAHGOUB, O. Effect of heat stress on respiratory rate, rectal temperature and blood chemistry in Omani and Australian Merino sheep. Small Ruminant Research, Amsterdam, v. 49, n. 2, p. 193-198, 2003. 
TABELEÃO, V. C.; DEL PINO, F. A. B.; GOULART, M. A.; WEISER, M. A.; SCHWEGLER, E.; MOURA, S. V.; SILVA, V. M.; ROOS, T. B.; GIL-TURNES, C.; GONZÁLEZ, F. H. D.; CORRÊA, M. N. Caracterização dos parâmetros ruminais e metabólicos de cordeiros mantidos em pastagem nativa. Ciência Animal Brasileira, Goiânia, v. 8, n. 4, p. 639-646, 2007.

VAN CLEEF, E. H.; PATINO, R. P.; NEIVA JUNIOR, A. P.; SERAFIM, R. S.; REGO, A. C.; GONCALVES, J. S. Distúrbios metabólicos por manejo alimentar inadequado em ruminantes: novos conceitos. Revista Colombiana Ciência Animais, Medellin, v. 1, n. 2, p. 319-325, 2009.
VAN KESSEL, J. A. S.; RUSSELL, J. B. The effect of $\mathrm{pH}$ on ruminal methanogenesis. FEMS Microbiology Ecology, Dubrovnik, v. 20, n. 4, p. 205-210, 1996.

WEINBERG, Z. G.; MUCK, R. E.; WEIMER, P. J.; CHEN, Y.; GAMBURG, M. Lactic acid bacteria used in inoculants for silage as probiotics for ruminants. Applied Biochemistry and Biotechnology, New York, v. 118, p. 1-9, 2004.

WILLIAMS, C. C.; CALMES, K, J.; FERNANDEZ, J. M.; STANLEY, C. C.; LOVEJOY, J. C.; BATEMAN II, H. G.; GENTRY, L. R.; GANTT, D. T.; HARDING, G. D. Glucose metabolism and insulin sensitivity in gulf coast native and suffolk ewes during late gestation and early lactation. Small Ruminant Research, Amsterdam, v. 54, n. 3, p. 167-171, 2004. 
